\title{
On Lifting Integer Variables in Minimal Inequalities
}

\author{
Amitabh Basu ${ }^{1,2}$, Manoel Campelo ${ }^{3,4}$, Michele Conforti ${ }^{5}$, \\ Gérard Cornuéjols ${ }^{1,6,7}$, Giacomo Zambelli ${ }^{5}$
}

November 18, 2009

\begin{abstract}
This paper contributes to the theory of cutting planes for mixed integer linear programs (MILPs). Minimal valid inequalities are well understood for a relaxation of an MILP in tableau form where all the nonbasic variables are continuous. In this paper we study lifting functions for the nonbasic integer variables starting from such minimal valid inequalities. We characterize precisely when the lifted coefficient is equal to the coefficient of the corresponding continuous variable in every minimal lifting. The answer is a nonconvex region that can be obtained as the union of convex polyhedra.
\end{abstract}

\section{Introduction}

There has been a renewed interest recently in the study of cutting planes for general mixed integer linear programs (MILPs) that cut off a basic solution of the linear programming relaxation. More precisely, consider a mixed integer linear set in which the variables are partitioned into a basic set $B$ and a nonbasic set $N$, and $K \subseteq B \cup N$ indexes the integer variables:

$$
\begin{array}{rlrl}
x_{i} & =f_{i}-\sum_{j \in N} a_{i j} x_{j} & & \text { for } i \in B \\
x & \geq 0 & & \\
x_{k} & \in \mathbb{Z} & \text { for } k \in K .
\end{array}
$$

Let $X$ be the relaxation of (1) obtained by dropping the nonnegativity restriction on all the basic variables $x_{i}, i \in B$. The convex hull of $X$ is the corner polyhedron introduced by Gomory [11] (see also [12]). Note that, for any $i \in B \backslash K$, the equation $x_{i}=f_{i}-$ $\sum_{j \in N} a_{i j} x_{j}$ can be removed from the formulation of $X$ since it just defines variable $x_{i}$. Therefore, throughout the paper, we will assume $B \subseteq K$, i.e. all basic variables are integer.

\footnotetext{
${ }^{1}$ Tepper School of Business, Carnegie Mellon University, Pittsburgh, PA 15213.

${ }^{2}$ Supported by a Mellon Fellowship and NSF grant CMMI0653419.

${ }^{3}$ Departamento de Estatística e Matemática Aplicada, Universidade Federal do Ceará, Brazil, mcampelo@lia.ufc.br

${ }^{4}$ Partially supported by CNPq Brazil

${ }^{5}$ Dipartimento di Matematica Pura e Applicata, Universitá di Padova, Via Trieste 63, 35121 Padova, Italy.

${ }^{6}$ LIF, Faculté des Sciences de Luminy, Université de Marseille, France.

${ }^{7}$ Supported by NSF grant CMMI0653419, ONR grant N00014-03-1-0133 and ANR grant ANR06-BLAN0375 .
} 
Andersen, Louveaux, Weismantel and Wolsey [1] studied the corner polyhedron when $|B|=2$ and $B=K$, i.e. all nonbasic variables are continuous. They give a complete characterization of the corner polyhedron using intersection cuts (Balas [2]) arising from splits, triangles and quadrilaterals. This very elegant result has been extended to $|B|>2$ and $B=K$ by showing a correspondence between minimal valid inequalities and maximal lattice-free convex sets [5], [7]. These results and their extensions [6], [10] are best described in an infinite model, which we motivate next.

A classical family of cutting planes for (1) is that of Gomory mixed integer cuts. For a given row of the tableau, the Gomory mixed integer cut is of the form $\sum_{j \in N \backslash K} \psi\left(a_{i j}\right) x_{j}+$ $\sum_{j \in N \cap K} \pi\left(a_{i j}\right) x_{j} \geq 1$ where $\psi$ and $\pi$ are functions given by simple formulas. A nice feature of the Gomory mixed integer cut is that, for fixed $f_{i}$, the same functions $\psi, \pi$ are used for any possible choice of the $a_{i j} \mathrm{~s}$ in (1). It is well known that the Gomory mixed integer cuts are also valid for $X$. More generally, let $a^{j}$ be the vector with entries $a_{i j}, i \in B$; we are interested in pairs $(\psi, \pi)$ of functions such that the inequality $\sum_{j \in N \backslash K} \psi\left(a^{j}\right) x_{j}+\sum_{j \in N \cap K} \pi\left(a^{j}\right) x_{j} \geq 1$ is valid for $X$ for any possible choice of the nonbasic coefficients $a_{i j}$. Since we are interested in nonredundant inequalities, we can assume that the function $(\psi, \pi)$ is pointwise minimal. While a general characterization of minimal valid functions seems hopeless (see for example [4]), when $N \cap K=\emptyset$ the minimal valid functions $\psi$ are well understood in terms of maximal lattice-free convex sets, as already mentioned. Starting from such a minimal valid function $\psi$, an interesting question is how to generate a function $\pi$ such that $(\psi, \pi)$ is valid and minimal. Recent papers [8], [9] study when such a function $\pi$ is unique. Here we prove a theorem that generalizes and unifies results from these two papers.

In order to formalize the concept of valid function $(\psi, \pi)$, we introduce the following infinite model. In the setting below, we also allow further linear constraints on the basic variables. Let $S$ be the set of integral points in some rational polyhedron in $\mathbb{R}^{n}$ such that $\operatorname{dim}(S)=n$ (for example, $S$ could be the set of nonnegative integer points). Let $f \in \mathbb{R}^{n} \backslash S$. Consider the following semi-infinite relaxation of (1), introduced in [10].

$$
\begin{aligned}
x= & f+\sum_{r \in \mathbb{R}^{n}} r s_{r}+\sum_{r \in \mathbb{R}^{n}} r y_{r}, \\
& x \in S, \\
& s_{r} \in \mathbb{R}_{+}, \forall r \in \mathbb{R}^{n}, \\
& y_{r} \in \mathbb{Z}_{+}, \forall r \in \mathbb{R}^{n}, \\
& s, y \text { have finite support }
\end{aligned}
$$

where the nonbasic continuous variables have been renamed $s$ and the nonbasic integer variables have been renamed $y$. Given two functions $\psi, \pi: \mathbb{R}^{n} \rightarrow \mathbb{R},(\psi, \pi)$ is said to be valid for (2) if the inequality $\sum_{r \in \mathbb{R}^{n}} \psi(r) s_{r}+\sum_{r \in \mathbb{R}^{n}} \pi(r) y_{r} \geq 1$ holds for every $(x, s, y)$ satisfying (2). We also consider the semi-infinite model where we only have continuous nonbasic variables.

$$
\begin{aligned}
x= & f+\sum_{r \in \mathbb{R}^{n}} r s_{r} \\
& x \in S, \\
& s_{r} \in \mathbb{R}_{+}, \forall r \in \mathbb{R}^{n}, \\
& s \text { has finite support. }
\end{aligned}
$$


A function $\psi: \mathbb{R}^{n} \rightarrow \mathbb{R}$ is said to be valid for (3) if the inequality $\sum_{r \in \mathbb{R}^{n}} \psi(r) s_{r} \geq 1$ holds for every $(x, s)$ satisfying (3). Given a valid function $\psi$ for (3), a function $\pi$ is a lifting of $\psi$ if $(\psi, \pi)$ is valid for (2). One is interested only in (pointwise) minimal valid functions, since non-minimal ones are implied by some minimal valid function. If $\psi$ is a minimal valid function for (3) and $\pi$ is a lifting of $\psi$ such that $(\psi, \pi)$ is a minimal valid function for (2) then we say that $\pi$ is a minimal lifting of $\psi$.

While minimal valid functions for (3) have a simple characterization [6], minimal valid functions for (2) are not well understood. A general idea to derive minimal valid functions for (2) is to start from some minimal valid function $\psi$ for (3), and construct a minimal lifting $\pi$ of $\psi$. While there is no general technique to compute such minimal lifting $\pi$, it is known that there exists a region $R_{\psi}$, containing the origin in its interior, where $\psi$ coincides with $\pi$ for any minimal lifting $\pi$. This latter fact was observed by Dey and Wolsey [9] for the case of $S=\mathbb{Z}^{2}$ and by Conforti, Cornuéjols and Zambelli [8] for the general case. Furthermore, it is remarked in [8] and [10] that, if $\pi$ is a minimal lifting of $\psi$, then $\pi(r)=\pi\left(r^{\prime}\right)$ for every $r, r^{\prime} \in \mathbb{R}^{n}$ such that $r-r^{\prime} \in \mathbb{Z}^{n} \cap \operatorname{lin}(\operatorname{conv}(S))$. Therefore the coefficients of any minimal lifting $\pi$ are uniquely determined in the region $R_{\psi}+\left(\mathbb{Z}^{n} \cap \operatorname{lin}(\operatorname{conv}(S))\right)$. In particular, whenever translating $R_{\psi}$ by integer vectors in $\operatorname{lin}(\operatorname{conv}(S))$ covers $\mathbb{R}^{n}, \psi$ has a unique minimal lifting. The purpose of this paper is to give a precise description of the region $R_{\psi}$.

To state our main result, we need to explain the characterization of minimal valid functions for (3). We say that a convex set $B \subseteq \mathbb{R}^{n}$ is $S$-free if $B$ does not contain any point of $S$ in its interior. A set $B$ is a maximal $S$-free convex set if it is an $S$-free convex set that is not properly contained in any $S$-free convex set. It was proved in [6] that maximal $S$-free convex sets are polyhedra containing a point of $S$ in the relative interior of each facet.

Given an $S$-free polyhedron $B \subseteq \mathbb{R}^{n}$ containing $f$ in its interior, $B$ can be uniquely written in the form

$$
B=\left\{x \in \mathbb{R}^{n}: a_{i}(x-f) \leq 1, i \in I\right\},
$$

where $I$ is a finite set of indices and $a_{i}(x-f) \leq 1$ is facet-defining for $B$ for every $i \in I$.

Let $\psi_{B}: \mathbb{R}^{n} \rightarrow \mathbb{R}$ be the function defined by

$$
\psi_{B}(r)=\max _{i \in I} a_{i} r, \quad \forall r \in \mathbb{R}^{n} .
$$

Note in particular that, since maximal $S$-free convex sets are polyhedra, the above function is defined for all maximal $S$-free convex sets $B$.

Theorem 1. [6] Let $\psi$ be a minimal valid function for (3). Then the set

$$
B_{\psi}:=\left\{x \in \mathbb{R}^{n} \mid \psi(x-f) \leq 1\right\}
$$

is a maximal $S$-free convex set containing $f$ in its interior, and $\psi=\psi_{B_{\psi}}$. Conversely, if $B$ is a maximal $S$-free convex set containing $f$ in its interior, then $\psi_{B}$ is a minimal valid function for (3).

We are now ready to state the main result of the paper. Given a minimal valid function $\psi$ for (3), by Theorem $1 B_{\psi}$ is a maximal $S$-free convex set containing $f$ in its interior, thus 
it can be uniquely written as $B_{\psi}=\left\{x \in \mathbb{R}^{n} \mid a_{i}(x-f) \leq 1, i \in I\right\}$. For every $r \in \mathbb{R}^{n}$, let $I(r)=\left\{i \in I \mid \psi(r)=a_{i} r\right\}$. Given $x \in S$, let

$$
R(x):=\left\{r \in \mathbb{R}^{n} \mid I(r) \supseteq I(x-f) \text { and } I(x-f-r) \supseteq I(x-f)\right\} .
$$

We define

$$
R_{\psi}:=\bigcup_{x \in S \cap B_{\psi}} R(x)
$$

Theorem 2. Let $\psi$ be a minimal valid function for (3). If $\pi$ is a minimal lifting of $\psi$, then $\pi(r)=\psi(r)$ for every $r \in R_{\psi}$.

Conversely, for every $\bar{r} \notin R_{\psi}$, there exists a lifting $\pi$ of $\psi$ such that $\pi(\bar{r})<\psi(\bar{r})$.

Figure 1 illustrates the region $R_{\psi}$ for several examples. We conclude the introduction presenting a different characterization of the regions $R(x)$.

Proposition 3. Let $\psi$ be a minimal valid function for (3), and let $x \in S$. Then $R(x)=\{r \in$ $\left.\mathbb{R}^{n} \mid \psi(r)+\psi(x-f-r)=\psi(x-f)\right\}$.

Proof. We can uniquely write $B_{\psi}=\left\{x \in \mathbb{R}^{n} \mid a_{i}(x-f) \leq 1, i \in I\right\}$. Let $h \in I(x-f)$. Then $\psi(x-f)=a_{h}(x-f)=a_{h} r+a_{h}(x-f-r) \leq \max _{i \in I} a_{i} r+\max _{i \in I} a_{i}(x-f-r)=\psi(r)+\psi(x-f-r)$.

In the above expression, equality holds if and only if $h \in I(r)$ and $h \in I(x-f-r)$.

\section{Minimum lifting coefficient of a single variable}

Given $r^{*} \in \mathbb{R}^{n}$, we consider the set of solutions to

$$
\begin{aligned}
x= & f+\sum_{r \in \mathbb{R}^{n}} r s_{r}+r^{*} y_{r^{*}} \\
& x \in S \\
& s \geq 0 \\
& y_{r^{*}} \geq 0, y_{r^{*}} \in \mathbb{Z} \\
& s \text { has finite support. }
\end{aligned}
$$

Given a minimal valid function $\psi$ for (3) and scalar $\lambda$, we say that the inequality $\sum_{r \in \mathbb{R}^{n}} \psi(r) s_{r}+$ $\lambda y_{r^{*}} \geq 1$ is valid for (4) if it holds for every $\left(x, s, y_{r^{*}}\right)$ satisfying (4). We denote by $\psi^{*}\left(r^{*}\right)$ the minimum value of $\lambda$ for which $\sum_{r \in \mathbb{R}^{n}} \psi(r) s_{r}+\lambda y_{r^{*}} \geq 1$ is valid for (4).

We observe that, for any lifting $\pi$ of $\psi$, we have

$$
\psi^{*}\left(r^{*}\right) \leq \pi\left(r^{*}\right)
$$

Indeed, $\sum_{r \in \mathbb{R}^{n}} \psi(r) s_{r}+\pi\left(r^{*}\right) y_{r^{*}} \geq 1$ is valid for (4), since, for any $\left(\bar{s}, \bar{y}_{r^{*}}\right)$ satisfying (4), the vector $(\bar{s}, \bar{y})$, defined by $\bar{y}_{r}=0$ for all $r \in \mathbb{R}^{n} \backslash\left\{r^{*}\right\}$, satisfies (2).

Moreover, the following fact was shown in [8]. 


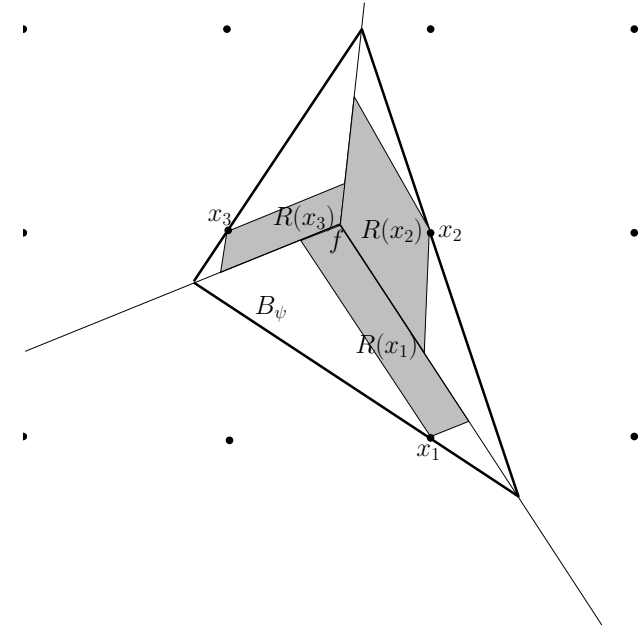

(a) A maximal $\mathbb{Z}^{2}$-free triangle with three integer points

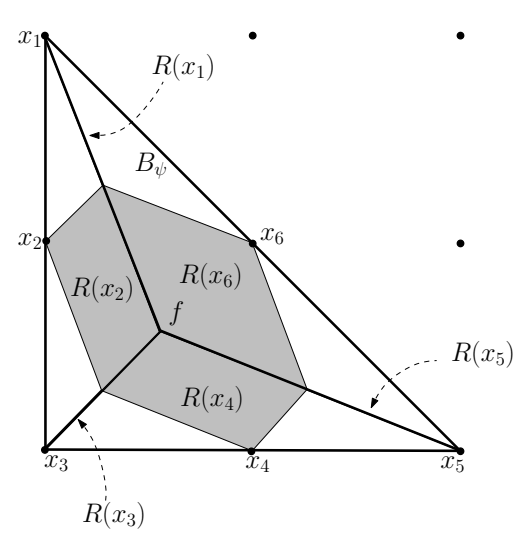

(b) A maximal $\mathbb{Z}^{2}$-free triangle with integer vertices

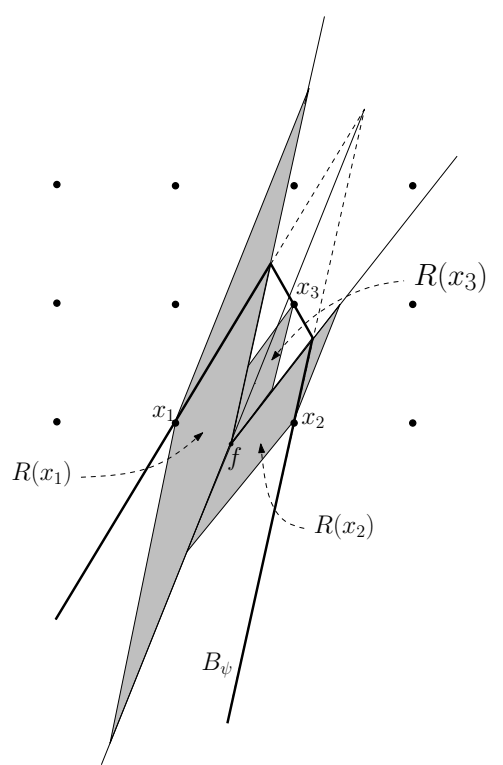

(d) A truncated wedge

(c) A wedge

Figure 1: Regions $R(x)$ for some maximal $S$-free convex sets in the plane. The thick dark line indicates the boundary of $B_{\psi}$. For a particular $x$, the dark gray regions denote $R(x)$. The jagged lines in a region indicate that it extends to infinity. For example, in Figure 1(c), $R\left(x_{1}\right)$ is the strip between lines $l_{1}$ and $l$. Figure $1(\mathrm{~b})$ shows an example where $R(x)$ is full-dimensional for $x_{2}, x_{4}, x_{6}$, but is not full-dimensional for $x_{1}, x_{3}, x_{5}$. 
Lemma 4. If $\psi$ is a minimal valid function for (3) and $\pi$ is a minimal lifting of $\psi$, then $\pi \leq \psi$.

So we have the following relation for every minimal lifting $\pi$ of $\psi$ :

$$
\psi^{*}(r) \leq \pi(r) \leq \psi(r) \quad \text { for all } r \in R^{n} .
$$

In general $\psi^{*}$ is not a lifting of $\psi$, but if it is, then the above relation implies that it is the unique minimal lifting of $\psi$.

Remark 5. For any $r \in \mathbb{R}^{n}$ such that $\psi^{*}(r)=\psi(r)$, we have $\pi(r)=\psi(r)$ for every minimal lifting $\pi$ of $\psi$. Conversely, if $\psi^{*}\left(r^{*}\right)<\psi\left(r^{*}\right)$ for some $r^{*} \in \mathbb{R}^{n}$, then there exists some lifting $\pi$ of $\psi$ such that $\pi\left(r^{*}\right)<\psi\left(r^{*}\right)$.

Proof. The first part follows from $\psi^{*} \leq \pi \leq \psi$. For the second part, given $r^{*} \in \mathbb{R}^{n}$ such that $\psi^{*}\left(r^{*}\right)<\psi\left(r^{*}\right)$, we can define $\pi$ by $\pi\left(r^{*}\right)=\psi^{*}\left(r^{*}\right)$ and $\pi(r)=\psi(r)$ for all $r \in \mathbb{R}^{n}, r \neq r^{*}$. Since $\psi$ is valid for (3), it follows by the definition of $\psi^{*}\left(r^{*}\right)$ that $\pi$ is a lifting of $\psi$.

By the above remark, in order to prove Theorem 2 we need to show that $R_{\psi}=\{r \in$ $\left.\mathbb{R}^{n} \mid \psi(r)=\psi^{*}(r)\right\}$. We will need the following results.

Theorem 6. [6] A full-dimensional convex set $B$ is a maximal $S$-free convex set if and only if it is a polyhedron such that $B$ does not contain any point of $S$ in its interior and each facet of $B$ contains a point of $S$ in its relative interior. Furthermore if $B \cap \operatorname{conv}(S)$ has nonempty interior, $\operatorname{lin}(B)$ contains $\operatorname{rec}(B \cap \operatorname{conv}(S))$.

Remark 7. The proof of Theorem 6 in [6] implies the following. Given a maximal $S$-free convex set $B$, there exists $\delta>0$ such that there is no point of $S \backslash B$ at distance less than $\delta$ from $B$.

Let $r^{*} \in \mathbb{R}^{n}$. Given a maximal $S$-free convex set $B=\left\{x \in \mathbb{R}^{n} \mid a_{i}(x-f) \leq 1, i \in I\right\}$, for any $\lambda \in \mathbb{R}$, we define the set $B(\lambda) \subset \mathbb{R}^{n+1}$ as follows

$$
B(\lambda)=\left\{\left(\begin{array}{c}
x \\
x_{n+1}
\end{array}\right) \in \mathbb{R}^{n+1} \mid a_{i}(x-f)+\left(\lambda-a_{i} r^{*}\right) x_{n+1} \leq 1, i \in I\right\} .
$$

Theorem 8. $\quad$ 87 Let $r^{*} \in \mathbb{R}^{n}$. Given a maximal $S$-free convex set $B$, let $\psi=\psi_{B}$. Given $\lambda \in \mathbb{R}$, the inequality $\sum_{r \in \mathbb{R}^{n}} \psi(r) s_{r}+\lambda y_{r^{*}} \geq 1$ is valid for (4) if and only if $B(\lambda)$ is $\left(S \times \mathbb{Z}_{+}\right)$free.

Remark 9. Let $r^{*} \in \mathbb{R}^{n}$, and let $B$ be a maximal $S$-free convex set. For every $\lambda$ such that $B(\lambda)$ is $\left(S \times \mathbb{Z}_{+}\right)$-free, $B(\lambda)$ is maximal $\left(S \times \mathbb{Z}_{+}\right)$-free.

Proof. Since $B$ is a maximal $S$-free convex set, then by Theorem 6 each facet of $B$ contains a point $\bar{x}$ of $S$ in its relative interior. Therefore the corresponding facet of $B(\lambda)$ contains the point $\left(\begin{array}{l}\bar{x} \\ 0\end{array}\right)$ in its relative interior. If $B(\lambda)$ is $\left(S \times \mathbb{Z}_{+}\right)$-free, by Theorem 6 it is a maximal $\left(S \times \mathbb{Z}_{+}\right)$-free convex set. 


\section{Characterizing the region $R_{\psi}$}

Next we state and prove a theorem that characterizes when $\psi^{*}\left(r^{*}\right)=\psi\left(r^{*}\right)$. The main result of this paper, Theorem 2, will then follow easily.

Theorem 10. Given a maximal $S$-free convex set $B$, let $\psi=\psi_{B}$. Given $r^{*} \in \mathbb{R}^{n}$, the following are equivalent:

(i) $\psi^{*}\left(r^{*}\right)=\psi\left(r^{*}\right)$.

(ii) There exists a point $\bar{x} \in S$ such that $\left(\begin{array}{l}\bar{x} \\ 1\end{array}\right) \in B\left(\psi\left(r^{*}\right)\right)$.

Proof. Let $\lambda^{*}=\psi\left(r^{*}\right)$. Note that the inequality $\sum_{r \in \mathbb{R}^{n}} \psi(r) s_{r}+\lambda^{*} y_{r^{*}} \geq 1$ is valid for (4). Thus, it follows from Theorem 8 that $B\left(\lambda^{*}\right)$ is $S \times \mathbb{Z}_{+}$-free .

We first show that (ii) implies (i). Assume there exists $\bar{x} \in S$ such that $\left(\begin{array}{l}\bar{x} \\ 1\end{array}\right) \in B\left(\lambda^{*}\right)$. Then, for every $\epsilon>0,\left(\begin{array}{l}\bar{x} \\ 1\end{array}\right)$ is in the interior of $B\left(\lambda^{*}-\epsilon\right)$, because $a_{i}(\bar{x}-f)+\left(\lambda-\varepsilon-a_{i} r^{*}\right) \leq 1-\varepsilon<1$ for all $i \in I$. Theorem 8 then implies that $\psi^{*}\left(r^{*}\right)=\lambda^{*}$.

Next we show that (i) implies (ii). Assume that $\psi^{*}\left(r^{*}\right)=\psi\left(r^{*}\right)=\lambda^{*}$. We recall that $\lambda^{*}=\max _{i \in I} a_{i} r^{*}$.

Note that, if $a_{i} r^{*}=\lambda^{*}$ for all $i \in I$, then $B\left(\lambda^{*}\right)=B \times \mathbb{R}$, so given any point $\bar{x}$ in $B \cap S$, $\left(\begin{array}{l}\bar{x} \\ 1\end{array}\right)$ is in $B\left(\lambda^{*}\right)$. Thus we assume that there exists an index $h$ such that $a_{h} r^{*}<\lambda^{*}$.

By Remark $9, B\left(\lambda^{*}\right)$ is maximal $\left(S \times \mathbb{Z}_{+}\right)$-free. Theorem 6 implies the following,

a) $\operatorname{rec}(B \cap \operatorname{conv}(S)) \subseteq \operatorname{lin}(B)$,

b) $\operatorname{rec}\left(B\left(\lambda^{*}\right) \cap \operatorname{conv}\left(S \times \mathbb{Z}_{+}\right)\right) \subseteq \operatorname{lin}\left(B\left(\lambda^{*}\right)\right)$.

Claim 1. $\operatorname{rec}\left(B\left(\lambda^{*}\right) \cap \operatorname{conv}\left(S \times \mathbb{Z}_{+}\right)\right)=\operatorname{rec}(B \cap \operatorname{conv}(S)) \times\{0\}$.

Let $\left(\begin{array}{c}\bar{r} \\ \bar{r}_{n+1}\end{array}\right) \in \operatorname{rec}\left(B\left(\lambda^{*}\right) \cap \operatorname{conv}\left(S \times \mathbb{Z}_{+}\right)\right)$. Note that $\operatorname{rec}\left(\operatorname{conv}\left(S \times \mathbb{Z}_{+}\right)\right)=\operatorname{rec}(\operatorname{conv}(S)) \times \mathbb{Z}_{+}$, thus $\bar{r} \in \operatorname{rec}(\operatorname{conv}(S))$ and $\bar{r}_{n+1} \geq 0$. We only need to show that $\bar{r}_{n+1}=0$.

By b), $\left(\begin{array}{c}\bar{r} \\ \bar{r}_{n+1}\end{array}\right)$ satisfies

$$
a_{i} \bar{r}+\left(\lambda^{*}-a_{i} r^{*}\right) \bar{r}_{n+1}=0, \quad i \in I .
$$

Since $\lambda^{*}-a_{i} r^{*} \geq 0$ and $\bar{r}_{n+1} \geq 0$,

$$
a_{i} \bar{r} \leq 0, \quad i \in I
$$

therefore $\bar{r} \in \operatorname{rec}(B)$. Thus $\bar{r} \in \operatorname{rec}(B \cap \operatorname{conv}(S))$ which, by a), is contained in $\operatorname{lin}(B)$. This implies

$$
a_{i} \bar{r}=0, \quad i \in I .
$$

It follows from the above and from (6) that $\left(\lambda^{*}-a_{i} r^{*}\right) \bar{r}_{n+1}=0$ for $i \in I$. Since $\lambda^{*}-a_{h} r^{*}>0$ for some index $h$, it follows that $\bar{r}_{n+1}=0$. This concludes the proof of Claim 1 .

Claim 2. There exists $\bar{\varepsilon}>0$ such that $\operatorname{rec}\left(B\left(\lambda^{*}-\varepsilon\right) \cap \operatorname{conv}\left(S \times \mathbb{Z}_{+}\right)\right)=\operatorname{rec}(B \cap \operatorname{conv}(S)) \times\{0\}$ for every $\varepsilon \in[0, \bar{\varepsilon}]$. 
Since $\operatorname{conv}(S)$ is a rational polyhedron, $S=\left\{x \in \mathbb{R}^{n} \mid C x \leq d\right\}$ for some rational matrix $(C, d)$. By Claim 1, there is no vector $\left(\begin{array}{l}r \\ 1\end{array}\right)$ in $\operatorname{rec}\left(B\left(\lambda^{*}\right) \cap \operatorname{conv}\left(S \times \mathbb{Z}_{+}\right)\right)$. Thus the system

$$
\begin{aligned}
a_{i} r+\left(\lambda^{*}-a_{i} r^{*}\right) & \leq 0, \quad i \in I \\
C r & \leq 0
\end{aligned}
$$

is infeasible. By Farkas Lemma, there exist scalars $\mu_{i} \geq 0, i \in I$ and a nonnegative vector $\gamma$ such that

$$
\begin{aligned}
\sum_{i \in I} \mu_{i} a_{i}+\gamma C & =0 \\
\lambda^{*}\left(\sum_{i \in I} \mu_{i}\right)-\left(\sum_{i \in I} \mu_{i} a_{i}\right) r^{*} & >0 .
\end{aligned}
$$

This implies that there exists some $\bar{\varepsilon}>0$ such that for all $\varepsilon \leq \bar{\varepsilon}$,

$$
\begin{aligned}
\sum_{i \in I} \mu_{i} a_{i}+\gamma C & =0 \\
\left(\lambda^{*}-\varepsilon\right)\left(\sum_{i \in I} \mu_{i}\right)-\left(\sum_{i \in I} \mu_{i} a_{i}\right) r^{*} & >0,
\end{aligned}
$$

thus the system

$$
\begin{aligned}
a_{i} r+\left(\lambda^{*}-\varepsilon-a_{i} r^{*}\right) & \leq 0, \quad i \in I \\
C r & \leq 0
\end{aligned}
$$

is infeasible. This implies that $\operatorname{rec}\left(B\left(\lambda^{*}-\varepsilon\right) \cap \operatorname{conv}\left(S \times \mathbb{Z}_{+}\right)\right)=\operatorname{rec}(B \cap \operatorname{conv}(S)) \times\{0\}$.

Claim 3. $B\left(\lambda^{*}\right)$ contains a point $\left(\begin{array}{c}\bar{x} \\ \bar{x}_{n+1}\end{array}\right) \in S \times \mathbb{Z}_{+}$such that $\bar{x}_{n+1}>0$.

By Claim 2, there exists $\bar{\varepsilon}$ such that, for every $\varepsilon \in[0, \bar{\varepsilon}], \operatorname{rec}\left(B\left(\lambda^{*}-\varepsilon\right) \cap \operatorname{conv}\left(S \times \mathbb{Z}_{+}\right)\right)=$ $\operatorname{rec}(B \cap \operatorname{conv}(S)) \times\{0\}$. This implies that there exists a scalar $M$ such that, for every $\varepsilon \in[0, \bar{\varepsilon}]$ and every point $\left(\begin{array}{c}\bar{x} \\ \bar{x}_{n+1}\end{array}\right) \in B\left(\lambda^{*}-\varepsilon\right) \cap\left(S \times \mathbb{Z}_{+}\right)$, it follows $\bar{x}_{n+1} \leq M$.

Remark 7 and Remark 9 imply that there exists $\delta>0$ such that, for every $\left(\begin{array}{c}\bar{x} \\ \bar{x}_{n+1}\end{array}\right) \in$ $\left(S \times \mathbb{Z}_{+}\right) \backslash B\left(\lambda^{*}\right)$, there exists $h \in I$ such that $a_{h}(\bar{x}-f)+\left(\lambda^{*}-a_{h} r^{*}\right) \bar{x}_{n+1} \geq 1+\delta$. Choose $\varepsilon>0$ such that $\varepsilon \leq \bar{\varepsilon}$ and $\varepsilon M \leq \delta$.

Since $\psi^{*}\left(r^{*}\right)=\lambda^{*}$, by Theorem $8, B\left(\lambda^{*}-\varepsilon\right)$ has a point $\left(\begin{array}{c}\bar{x} \\ \bar{x}_{n+1}\end{array}\right) \in S \times \mathbb{Z}_{+}$in its interior. Thus $a_{i}(\bar{x}-f)+\left(\lambda^{*}-\varepsilon-a_{i} r^{*}\right) \bar{x}_{n+1}<1, i \in I$.

We show that $\left(\begin{array}{c}\bar{x} \\ \bar{x}_{n+1}\end{array}\right)$ is also in $B\left(\lambda^{*}\right)$. Suppose not. Then, by our choice of $\delta$, there exists $h \in I$ such that $a_{h}(\bar{x}-f)+\left(\lambda^{*}-a_{h} r^{*}\right) \bar{x}_{n+1} \geq 1+\delta$. By our choice of $M$ and $\varepsilon$,

$1+\delta \leq a_{h}(\bar{x}-f)+\left(\lambda^{*}-a_{h} r^{*}\right) \bar{x}_{n+1} \leq a_{h}(\bar{x}-f)+\left(\lambda^{*}-\varepsilon-a_{h} r^{*}\right) \bar{x}_{n+1}+\varepsilon M<1+\varepsilon M \leq 1+\delta$, a contradiction.

Hence $\left(\begin{array}{c}\bar{x} \\ \bar{x}_{n+1}\end{array}\right)$ is in $B\left(\lambda^{*}\right)$. Since $B$ is $S$-free and $B\left(\lambda^{*}-\varepsilon\right) \cap\left(\mathbb{R}^{n} \times\{0\}\right)=B \times\{0\}$, it follows that $B\left(\lambda^{*}-\varepsilon\right)$ does not contain any point of $S \times\{0\}$ in its interior. Thus $\bar{x}_{n+1}>0$. This concludes the proof of Claim 3. 
By the previous claim, $B\left(\lambda^{*}\right)$ contains a point $\left(\begin{array}{c}\bar{x} \\ \bar{x}_{n+1}\end{array}\right) \in S \times \mathbb{Z}_{+}$such that $\bar{x}_{n+1}>0$. Note that $B\left(\lambda^{*}\right)$ contains $\left(\begin{array}{l}\bar{x} \\ 1\end{array}\right)$, since

$$
a_{i}(\bar{x}-f)+\left(\lambda^{*}-a_{i} r^{*}\right) \leq a_{i}(\bar{x}-f)+\left(\lambda^{*}-a_{i} r^{*}\right) \bar{x}_{n+1} \leq 1, \quad i \in I
$$

since $\lambda^{*}-a_{i} r^{*} \geq 0, i \in I$.

Corollary 11. Let $\psi$ be a minimal valid function for (3). Then $\psi^{*}\left(r^{*}\right)=\psi\left(r^{*}\right)$ if and only if there exists $\bar{x} \in S$ such that

$$
\psi\left(r^{*}\right)+\psi\left(\bar{x}-f-r^{*}\right)=1 .
$$

Proof. We first show that, if there exist $\bar{x} \in S$ satisfying (7), then $\psi^{*}\left(r^{*}\right)=\psi\left(r^{*}\right)$. Indeed, since $\sum_{r \in \mathbb{R}^{n}} \psi(r) s_{r}+\psi^{*}\left(r^{*}\right) y_{r^{*}} \geq 1$ is valid for (4),

$$
1 \leq \psi^{*}\left(r^{*}\right)+\psi\left(\bar{x}-f-r^{*}\right) \leq \psi\left(r^{*}\right)+\psi\left(\bar{x}-f-r^{*}\right)=1 .
$$

We show the converse. Since $\psi$ is a valid function for $(3), \psi\left(\bar{x}-f-r^{*}\right)+\psi\left(r^{*}\right) \geq 1$. Since $\psi$ is a minimal valid function for (3), by Theorem 1 there exists a maximal $S$-free convex set $B$ such that $\psi=\psi_{B}$. Let $B_{\psi}=\left\{x \in \mathbb{R}^{n} \mid a_{i}(x-f) \leq 1, i \in I\right\}$.

Assume $\psi^{*}\left(r^{*}\right)=\psi\left(r^{*}\right)$. By Theorem 10, there exists a point $\bar{x} \in S$ such that $\left(\begin{array}{l}\bar{x} \\ 1\end{array}\right) \in$ $B\left(\psi\left(r^{*}\right)\right)$. Therefore

$$
a_{i}(\bar{x}-f)+\psi\left(r^{*}\right)-a_{i} r^{*} \leq 1, \quad i \in I .
$$

Thus

$$
\max _{i \in I} a_{i}\left(\bar{x}-f-r^{*}\right) \leq 1-\psi\left(r^{*}\right),
$$

which implies $\psi\left(\bar{x}-f-r^{*}\right)+\psi\left(r^{*}\right) \leq 1$. Hence $\psi\left(\bar{x}-f-r^{*}\right)+\psi\left(r^{*}\right)=1$.

Proof of Theorem 2. By Remark 5, we only need to show that $R_{\psi}=\left\{r \in \mathbb{R}^{n} \mid \psi(r)=\psi^{*}(r)\right\}$. For every $x \in S$, we have $\psi(x-f)=1$ if and only if $x \in S \cap B_{\psi}$. Therefore, by Proposition 3, $R(x)=\left\{r \in \mathbb{R}^{n} \mid \psi(r)+\psi(x-f-r)=\psi(x-f)=1\right\}$ if and only if $x \in S \cap B_{\psi}$. The latter fact and Corollary 11 imply that a vector $r \in \mathbb{R}^{n}$ satisfies $\psi^{*}(r)=\psi(r)$ if and only if $r \in R(x)$ for some $x \in S \cap B_{\psi}$. The statement now follows from the definition of $R_{\psi}$.

\section{Conclusion}

In this paper we give an exact characterization of the region where a minimal valid inequality $\psi$ and any minimal lifting $\pi$ of $\psi$ coincide. This was exhibited in Theorem 2, which generalizes results from [8] and [9] about liftings of minimal valid inequalities.

As already mentioned in the introduction, the following theorem was proved in [8].

Theorem 12. Let $\psi$ be a minimal valid function for (3). If $R_{\psi}+\left(\mathbb{Z}^{n} \cap \operatorname{lin}(\operatorname{conv}(S))\right)$ covers all of $\mathbb{R}^{n}$, then there exists a unique minimal lifting $\pi$ of $\psi$.

We conjecture that the converse also holds.

Conjecture 13. Let $\psi$ be a minimal valid function for (3). There exists a unique minimal lifting $\pi$ of $\psi$ if and only if $R_{\psi}+\left(\mathbb{Z}^{n} \cap \operatorname{lin}(\operatorname{conv}(S))\right)$ covers all of $\mathbb{R}^{n}$. 


\section{Acknowledgements}

The authors would like to thank Marco Molinaro for helpful discussions about the results presented in this paper.

\section{References}

[1] K. Andersen, Q. Louveaux, R. Weismantel and L. A. Wolsey, Cutting Planes from Two Rows of a Simplex Tableau, Proceedings of IPCO XII, Ithaca, New York (June 2007), Lecture Notes in Computer Science 4513, 1-15.

[2] E. Balas, Intersection Cuts - A New Type of Cutting Planes for Integer Programming, Operations Research 19 (1971), 19-39.

[3] A. Barvinok, A Course in Convexity, Graduate Studies in Mathematics, Vol. 54, American Mathematical Society, Providence, Rhode Island, 2002.

[4] A. Basu, M. Conforti, G. Cornuejols, G. Zambelli, A Counterexample to a Conjecture of Gomory and Johnson, manuscript (November 2008).

[5] A. Basu, M. Conforti, G. Cornuejols, G. Zambelli, Maximal Lattice-free Convex Sets in Linear Subspaces, manuscript (March 2009).

[6] A. Basu, M. Conforti, G. Cornuejols, G. Zambelli, Minimal Inequalities for an Infinite Relaxation of Integer Programs, manuscript (April 2009).

[7] V. Borozan, G. Cornuéjols, Minimal Valid Inequalities for Integer Constraints, technical report (July 2007), to appear in Mathematics of Operations Research.

[8] M. Conforti, G. Cornuejols, G. Zambelli, A Geometric Perspective on Lifting, manuscript (May 2009).

[9] S.S. Dey, L.A. Wolsey, Lifting Integer Variables in Minimal Inequalities corresponding to Lattice-Free Triangles, IPCO 2008, Bertinoro, Italy (May 2008), Lecture Notes in Computer Science 5035, 463-475.

[10] S.S. Dey, L.A. Wolsey, Constrained Infinite Group Relaxations of MIPs, manuscript (March 2009).

[11] R.E. Gomory, Some Polyhedra related to Combinatorial Problems, Linear Algebra and its Applications 2 (1969), 451-558.

[12] R.E. Gomory and E.L. Johnson, Some Continuous Functions Related to Corner Polyhedra, Part I, Mathematical Programming 3 (1972) 23-85.

[13] E.L. Johnson, On the Group Problem for Mixed Integer Programming, Mathematical Programming Study (1974) 137-179.

[14] A. Schrijver, Theory of Linear and Integer Programming, John Wiley \& Sons, New York (1986). 
[15] R.R. Meyer, On the Existence of Optimal Solutions to Integer and Mixed-Integer Programming Problems, Mathematical Programming 7 (1974) 223-235. 